INTERVENTIONAL CARDIOLOGY AND SURGERY

\title{
Thoracoscopic ASD closure is a reliable supplement for percutaneous treatment
}

\author{
F P Casselman, H Dom, B De Bruyne, Y Vermeulen, H Vanermen
}

Heart 2005;91:791-794. doi: 10.1136/hrt.2004.034041

See end of article for authors' affiliations .....................

Correspondence to: Dr Filip P Casselman, Department of Cardiovascular and Thoracic Surgery, OLV Clinic, Moorselbaan 164, 9300 Aalst, Belgium; filip. casselman@olvz-aalst.be

Accepted 15 July 2004
Objective: To determine the feasibility and effectiveness of endoscopic atrial septal defect (ASD) closure when percutaneous ASD closure is impossible or has failed.

Patients: Between March 1997 and February 2003, 74 patients (63\% female, mean (SD) age 44 (16) years) underwent an endoscopic ASD closure. Median preoperative New York Heart Association functional class was I. Clinical and echocardiographic follow up was obtained for all patients (mean (SD) 38 (19) months). Patients were assessed for scar aesthetics, procedure related pain, functional recovery, and overall patient satisfaction.

Results: ASD closure was successful in all patients (two primum ASD, 68 secundum ASD, four sinus venosus type). Patch repair was performed in $42 \%$. Mean aortic cross clamp and cardiopulmonary bypass times were 54 (24) minutes and 98 (35) minutes, respectively. There were no in-hospital deaths and no conversions to sternotomy. Complications included one iliac vein stenting, one femoral arterioplasty, two revisions for suspected bleeding, and seven cases of atrial fibrillation. Two patients required late reoperation: one for atrial thrombus and another for tricuspid regurgitation. Echocardiographic control confirmed complete ASD closure in 71 patients and a small residual shunt in three patients. Ninety three per cent of the patients were highly satisfied with very low procedure related pain and $97 \%$ felt they had an aesthetically pleasing scar.

Conclusion: Endoscopic ASD closure can be safely done with a high degree of patient satisfaction. It is now the authors' exclusive surgical approach whenever percutaneous treatment is not indicated or has failed.
$\mathrm{T}$ ranscatheter closure of secundum atrial septal defects (ASDs) is standard practice and excellent results have been reported with low early and late complication rates, both in low and higher risk patient groups. ${ }^{12}$ However, ostium primum and sinus venosus ASDs as well as large secundum defects with limited septal margins and fenestrated or aneurysmal interatrial septa are less suitable for percutaneous closure. ${ }^{3}$ In addition, surgery may be indicated for unsuccessful or complicated percutaneous closure of ASD or persistent foramen ovale. ${ }^{3-6}$ To avoid a median sternotomy and its related discomfort, we have been operating on these patients with a minimally invasive endoscopic technique. We report our experience with this technique.

\section{METHODS \\ Patients}

Between March 1997 and February 2003, 74 patients underwent endoscopic ASD closure. Mean (SD) age was 44 (16) years (range 10-74 years) and 63\% were female. All patients were referred by a cardiological team who judged the patient unsuitable for percutaneous closure. In three patients, surgical closure followed an unsuccessful percutaneous procedure. All patients who presented were accepted for the technique. All procedures were primary surgical procedures. Median preoperative New York Heart Association (NYHA) functional class was I (range I-IV) and the preoperative rhythm was sinus rhythm in 68 patients (92\%), atrial fibrillation in five patients $(7 \%)$, and pacemaker rhythm in one patient $(1 \%)$. The septal defect was a primum $(n=2$, $3 \%)$, secundum $(\mathrm{n}=68,92 \%)$, or sinus venosus $(\mathrm{n}=4$, $5 \%$ ) defect. Mean (SD) ASD defect size was 21.5 (11.2) mm (range 5.5-60 mm) and the mean (SD) preoperative ratio of pulmonary to systemic blood flow was $2.2(0.6)$. The left ventricular ejection fraction was normal in $78 \%$ of patients, mildly impaired in 19\%, and moderately impaired in the remainder $(3 \%)$. Right ventricular dilatation was present in 54 patients $(73 \%)$ and pulmonary hypertension in 37 patients $(50 \%)$. The mean (SD) preoperative systolic pulmonary artery pressure was 37 (10) $\mathrm{mm} \mathrm{Hg}$ (range 22-74 mm Hg).

Patients undergoing cardiac catheterisation preoperatively (usually above 40 years of age or in the presence of risk factors) also had an injection in the iliac arteries to detect atherosclerotic disease precluding the use of the endocardiopulmonary bypass system.

All patients were fully informed about the technique preoperatively and were able to choose a median sternotomy if that was their preference. However, none of them did so.

Postoperatively, all patients had a transthoracic echocardiogram. Patients were routinely seen at the outpatient clinic six weeks postoperatively and then referred to their cardiologist. Only patients undergoing patch closure were routinely anticoagulated for six weeks; patients in atrial fibrillation were permanently anticoagulated unless antiarrhythmic treatment was effective. The other patients did not routinely receive antiplatelet treatment.

\section{Operative technique}

The technique used is similar to that used for endoscopic mitral valve repair with some adaptations for ASD repair. ${ }^{7}$

The procedure is performed under general anaesthesia with a double lumen endotracheal tube and transoesophageal echocardiographic guidance. The anaesthesiologist places a percutaneous right jugular venous cannula to drain the superior body. The patient is then draped and the right femoral vessels are exposed through a $3 \mathrm{~cm}$ oblique groin incision. Next, a $4 \mathrm{~cm}$ working port is created in the right inframammary groove, usually in the fourth intercostal space. Another port is used for suction and $\mathrm{CO}_{2}$ insufflation. 


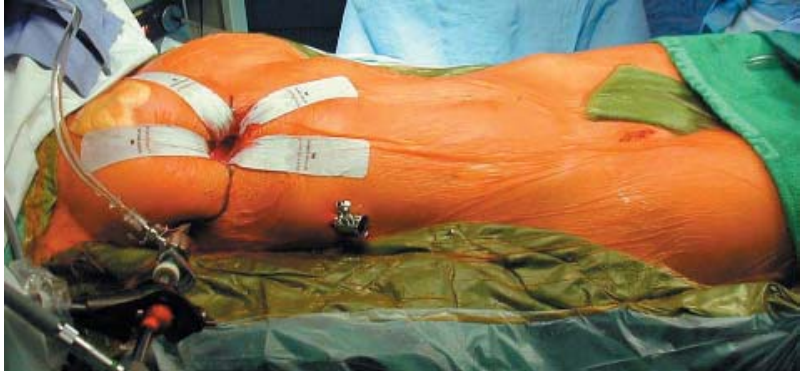

Figure 1 Operative setup. Note the central small working port through which the entire procedure is performed. The femoral vessels will be used to connect the extracorporeal circulation circuit. Reproduced with permission of the patient.

Long shafted instruments are a prerequisite to perform the operation. An endoscope provides visualisation through a separate port in the fourth intercostal space at the anterior axillary line (fig 1). Femoro-femoral extracorporeal circulation is used as well as an endoaortic balloon for endoluminal aortic clamping and antegrade cold crystalloid cardioplegia administration. Both caval veins are taped and the right atrium is opened. At this stage, a malpositioned device can be retrieved (fig 2) and the ASD is closed by either a direct or patch closure technique (fig 3).

Once the cardiopulmonary bypass is discontinued, the right chest and pericardium are drained. The pericardium is closed and all skin incisions are closed intracutaneously (fig 4).

\section{Follow up}

Data were collected retrospectively. All patients were either seen at the outpatient clinic or contacted by telephone for follow up. The latest transthoracic echocardiogram available was used to determine technical effectiveness.

In addition, patients were asked about the amount of pain they experienced at the time of the operation, how fast postoperatively they had regained professional or expected activity, whether they were pleased with the aesthetic result, and whether they would prefer this technique over standard median sternotomy if they had to choose again.

\section{Data analysis}

The design of the study was retrospective and data are expressed as the mean (SD). Data were analysed with the Statistica package release 5.1 (StatSoft, Tulsa, Oklahoma, USA). A two sided $\mathrm{p}<0.05$ was considered significant.

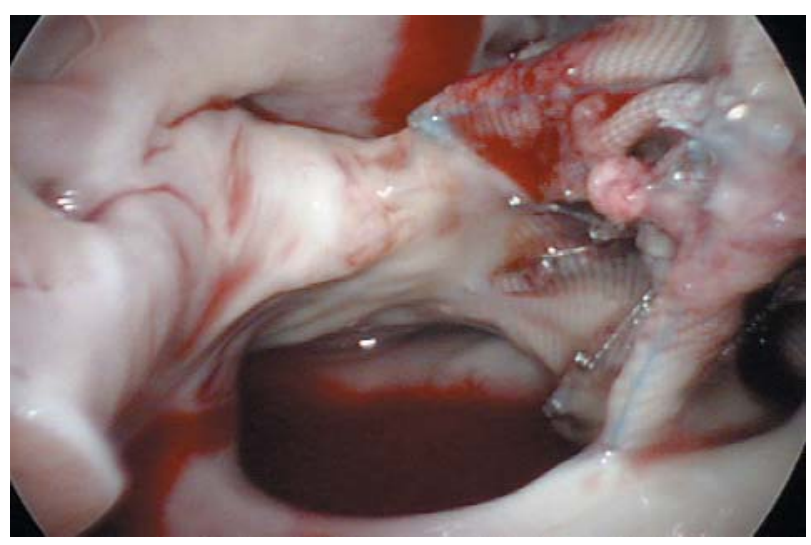

Figure 2 A malpositioned device will be removed. Reproduced with permission of the patient.

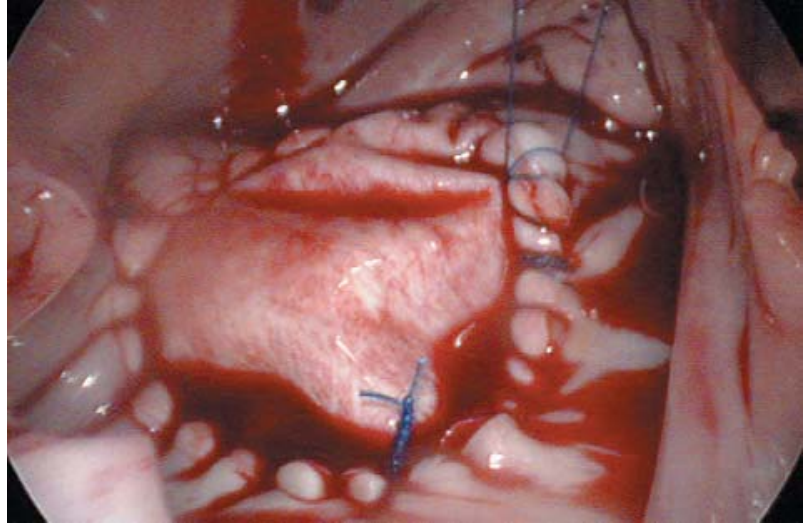

Figure 3 Thoracoscopic atrial septal defect repair with a bovine pericardial patch to close a type II defect. Reproduced with permission of the patient.

\section{RESULTS}

\section{Operative technique}

All procedures were successful and there were no conversions to median sternotomy. The visualisation of the operative field was extraordinary and permitted direct ASD closure in 45 patients $(61 \%)$ and patch repair in the remainder (29 patients, 39\%). Mean (SD) aortic cross clamp and cardiopulmonary bypass times were 54 (24) minutes (range 24-135 minutes) and 98 (35) minutes (range 30-198 minutes), respectively.

Intraoperative complications occurred in two patients: one patient required iliac vein stenting due to a laceration and in another patient the common femoral artery was stenosed after arterial cannula retrieval and closure of the puncture site. Consequently, a patch enlargement was performed.

\section{Postoperative follow up}

Mean (SD) postoperative chest tube output was 785 (769) ml (median $590 \mathrm{ml}$ ).

Mean (SD) postoperative intensive care unit stay was 24 (10) hours (range 5-70 hours) and postoperative hospital stay was 5 (2) days (range 3-12 days).

No patients died postoperatively and postoperative morbidity was low (table 1). Two patients were taken back for persistent bleeding. They were re-explored through the same working port. In one patient, the right atriotomy was oozing and in

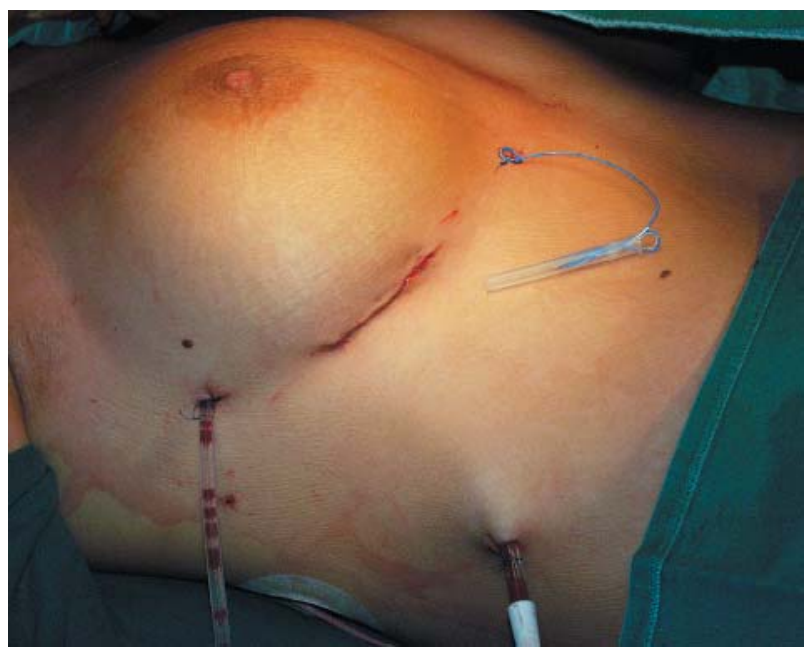

Figure 4 Closure after thoracoscopic atrial septal defect repair. Reproduced with permission of the patient. 
Table 1 Postoperative complications $(n=74)$

\begin{tabular}{ll}
\hline Type of complication & Number \\
\hline Revision for suspected bleeding & $2(3 \%)$ \\
New onset atrial fibrillation & $7(9 \%)$ \\
Pacemaker implantation & $3(4 \%)$ \\
Renal insufficiency & 0 \\
Peripheral ischaemic event & 0 \\
Deep venous thrombosis & 0 \\
Wound infection & 0 \\
Postoperative right pneumothorax & $4(5 \%)$ \\
\hline
\end{tabular}

another patient an intercostal artery was actively bleeding. Three patients required postoperative pacemaker implantation. One of them, however, already had a sick sinus syndrome preoperatively and it was decided to close the ASD first.

\section{Late follow up}

Mean (SD) patient follow up was 38 (19) months (range 273 months) and was $100 \%$ complete. The NYHA functional class at follow up was I in 72 patients and II in one (there was one late death) $(\mathrm{p}<0.001$ versus preoperatively). At follow up, 68 patients were in sinus rhythm, two were in atrial fibrillation, and three had pacemaker rhythm.

Post-hospital discharge follow up found one pulmonary embolus in a female patient three weeks postoperatively. Duplex sonography was negative for deep venous thrombosis. This patient underwent a primary closure of a secundum ASD and was not taking warfarin. Consequently, she was anticoagulated. Three patients developed a right groin cystic lymphangioma, which was punctured and further treated conservatively.

In addition, there were two repeat operations, one at six and another at 18 months postoperatively. The first patient was a 71 year old woman in very moderate condition who also had grade 2 tricuspid and mitral valve regurgitation preoperatively. She underwent primary closure of a secundum-type ASD. She initially did well but after five months progressively went into right heart failure with grade 4 tricuspid valve regurgitation and still grade 2 mitral valve regurgitation. She underwent tricuspid valve annuloplasty through median sternotomy six months after ASD repair. Postoperatively she remained in precarious condition and her mitral valve regurgitation deteriorated necessitating a third intervention for mitral valve repair six weeks later. However, she progressively went into multiple organ failure and died eight months after the first intervention.

The other patient had undergone a patch repair of a secundum-type ASD. On routine follow up 18 months postoperatively, a mass was seen in the right atrium at the junction with the inferior caval vein. The mass was removed by the same minimally invasive approach and appeared to be an old thrombus. The patient was prescribed lifelong anticoagulation and is currently well.

Follow up echocardiographic examination found a very small residual shunt in three patients. All of them are being treated conservatively. Eight patients had echocardiographic signs of mild pulmonary hypertension $(\mathrm{p}<0.001$ versus preoperatively).

\section{Patient satisfaction}

Of all patients, 93\% stated they experienced little to almost no procedure related pain. None of the remainder mentioned excessive procedure related pain.

Twenty six per cent of the patients were back at work or doing routine activities within four weeks postoperatively. Another 25\% were back at this activity level within eight weeks postoperatively.
Ninety seven per cent of the patients were extremely pleased with the cosmetic result of the procedure. In $44 \%$ of the patients, the scar was hardly visible any more.

Ninety three per cent of the patients said they would choose the same procedure if they were to face the same operation again.

\section{DISCUSSION}

Standard surgical ASD closure provides excellent results with a very low operative mortality and morbidity. ${ }^{8-11}$ However, cardiac surgery and medicine as a whole are strongly evolving in a minimally invasive direction. Moreover, the advent of transcatheter closing devices has substantially shifted the treatment of (secundum) ASDs from the surgeon towards the interventionalist. Excellent results have been reported with the use of these percutaneous devices. ${ }^{12}$ However, transcatheter closure is especially indicated for secundum-type ASD and has not yet conquered the primum and sinus venosustype ASDs. Besides, a very large secundum-type ASD, a defect with an insufficient rim to anchor the device, and a fenestrated or aneurysmal interatrial septum have also proved to be less optimal indications for transcatheter closure. ${ }^{13}$ Therefore, surgery certainly still has a place in the treatment of ASD closure, even in the secundum type. ${ }^{12}$

As an alternative to standard median sternotomy, several groups have reported their experience with right anterolateral thoracotomy as the surgical approach. Some have reported increased difficulty for complex $\mathrm{ASDs}^{13}{ }^{14}$ or increased phrenic nerve injury, ${ }^{15}$ whereas others have reported excellent results for all ASD types with a minimally invasive approach versus standard sternotomy. ${ }^{16-19}$ Our series supports the latter findings. Although the majority of our patient population underwent a secundum-type repair, the technique proved to be applicable to any type, with no operative mortality and low perioperative morbidity. Besides, surgical intervention was successful for all patients considered for the procedure. The only contraindications for the application of the presented technique are severe peripheral vascular disease (limiting safe femoral artery cannulation), severe right pleural adhesions (limiting right lung collapse and right heart exposure), presence of a pectus excavatum (dislocating the heart into the left chest), aortic regurgitation grade $>1$ (precluding the use of antegrade cardioplegia administration), and a dilated ascending aorta limiting correct positioning of the endoluminal aortic clamp. Obviously, the femoral artery needs to have a large enough diameter to permit the insertion of a 21 French arterial cannula. This explains why the presented technique is not applicable in young children, although an arterial access through the right iliac artery has been proposed in such instances. ${ }^{20}$

An advantage of the minimally invasive method is that fewer adhesions are created intraoperatively, since the dissection is much reduced. This is particularly important in young adults, since they may still be subjected to subsequent surgical interventions later in life. Reduced adhesions will render the subsequent procedure less hazardous.

Most reported minimally invasive ASD closure procedures were done with a limited right anterolateral thoracotomy.

Torracca and colleagues ${ }^{19}$ reported ASD closure with a robotic approach. Whether this method will prove to be superior to other minimally invasive approaches remains to be determined, since the six patients reported on all had straightforward secundum-type ASDs in contrast to the various types of ASD repair reported in the current series. Robotic ASD surgery is still in an early stage and we are curiously awaiting results of more complex repairs with the robotic approach.

The improved cosmetic result is obviously a major advantage of the technique presented. This probably explains 
why the patient satisfaction rate in the present series was so high. Similar results have been reported from other series. ${ }^{16-18}$ Certainly, the majority of the patients were women and consequently the surgical scar was nicely hidden by the breast. But male patients also very much appreciated our surgical approach. The current patient population is very much interested in the cosmetic result of the procedure, as long as operative risks do not increase. In addition, the pain related to the procedure can be very much limited (as shown by our patient survey). All of these factors probably explain why none of our patients would choose a median sternotomy if he or she had to face the procedure again. In our opinion, only the necessity of correcting additional pathological conditions other than mitral or tricuspid valve disease or the presence of contraindications to the current technique warrants the use of a median sternotomy to surgically correct an ASD of any type.

One may argue that only $51 \%$ of the patients were back at full professional activity within eight weeks postoperatively. This is obviously true but we evaluated this very strictly: full activity in our patient survey meant $100 \%$, not $80 \%$, for example. Besides, social security rules in Belgium permit patients to be professionally non-active for three months after cardiac surgery. Obviously, many patients want to respect this rule!

Overall, improved cosmesis, reduced operative pain, and a relatively fast rehabilitation in an economically active patient population are all important considerations and undoubtedly contributed to the very positive patient attitude towards this procedure.

\section{Conclusions}

The presented technique provides an optimal exposure permitting surgical correction of any type of ASD. The technique has no operative mortality so far and a low perioperative morbidity. It is our exclusive operative approach whenever percutaneous ASD closure is impossible or has failed.

\section{ACKNOWLEDGEMENTS}

The authors thank Karlien Meuleman for secretarial assistance.

\section{Authors' affiliations}

F P Casselman, H Dom, Y Vermeulen, H Vanermen, Department of Cardiovascular and Thoracic Surgery, OLV Clinic, Aalst, Belgium

B De Bruyne, Department of Cardiology, OLV Clinic

\section{REFERENCES}

1 Chessa M, Carminati M, Butera G, et al. Early and late complications associated with transcatheter occlusion of secundum atrial septal defect. J Am Coll Cardiol 2002;39:1061-5.

2 Suarez de Lezo J, Medina A, Romero M, et al. Effectiveness of percutaneous device occlusion for atrial septal defect in adult patients with pulmonary hypertension. Am Heart J 2002;144:877-80.

3 Austin EH. Transcatheter closure of atrial septal defects. J Thorac Cardiovasc Surg 2003; 125:S85-6.

4 Berdat PA, Chatterjee T, Pfammatter JP, et al. Surgical management of complications after transcatheter closure of an atrial septal defect or patent foramen ovale. J Thorac Cardiovasc Surg 2000;120:1034-9.

5 Fukahara K, Minami K, Reiss N, et al. Systemic allergic reaction to the percutaneous patent foramen ovale occluder. J Thorac Cardiovasc Surg 2003; 125:213-4

6 Borgermann J, Hakim K, Friedrich I, et al. Recurrent thromboembolic events after percutaneous device closure of patent foramen ovale. Interactive Cardiovasc Thorac Surg 2003;2:125-7.

7 Casselman F, Van Slycke S, Dom H, et al. Endoscopic mitral valve repair: feasible, reproducible and durable. J Thorac Cardiovasc Surg 2003; 125:273-82.

8 Murphy JG, Gersh BJ, McGoon MD, et al. Long-term outcome after surgical repair of isolated atrial septal defect: follow-up at 27-32 years. N Engl J Med 1990;323:1645-50

9 Horvath KA, Burke RP, Collins JJ Jr, et al. Surgical treatment of adult atrial septal defect: early and long-term results. J Am Coll Cardiol 1992;20:1156-9.

10 Galal MO, Wobst A, Halees Z, et al. Perioperative complications following surgical closure of atrial septal defect type II in 232 patients: a baseline study. Eur Heart J 1994;15:1381-4.

11 Pastorek JS, Allen HD, Davis JT. Current outcomes of surgical closure of secundum atrial septal defect. Am J Cardiol 1994;74:75-7.

12 Cowley CG, Lloyd TR, Bove EL, et al. Comparison of results of closure of secundum atrial septal defect by surgery versus Amplatzer septal occluder. Am J Cardiol 2001;88:589-91.

13 Massetti M, Babatasi G, Rossi A, et al. Operation for atrial septal defect through a right anterolateral thoracotomy: current outcome. Ann Thorac Surg 1996;62:1100-3.

14 Schmid FX, Wipperman CF, Hake U, et al. Surgical closure of atrial septal defects via right-sided thoracotomy: value with reference to the development of interventional closure techniques. Z Kardiol 1996;85:489-94.

15 Helps B-A, Ross-Russel RI, Dicks-Mireau C, et al. Phrenic nerve damage via a right thoracotomy in older children with secundum ASD. Ann Thorac Surg 1993:56:328-30.

16 Dabritz S, Sachweh J, Walter M, et al. Closure of atrial septal defects via limited right anterolateral thoracotomy as a minimal invasive approach in female patients. Eur J Cardiothorac Surg 1999;15:18-23.

17 Ryan WH, Cheirif J, Dewey TM, et al. Safety and efficacy of minimally invasive atrial septal defect closure. Ann Thorac Surg 2003;75:1532-4.

18 Doll N, Walther T, Falk V, et al. Secundum ASD closure using a right lateral minithoracotomy: five-year experience in 122 patients. Ann Thorac Surg 2003:75:1527-31.

19 Torracca L, Ismeno G, Alfieri O. Totally endoscopic computer-enhanced atrial septal defect closure in six patients. Ann Thorac Surg $2001 ; 72: 1354-7$

20 Grinda JM, Folliguet TA, Dervanian P, et al. Right anterolateral thoracotomy for repair of atrial septal defect. Ann Thorac Surg 1996;62:175-8. 